\title{
The mass and age of the first SONG target: the red giant $46 \mathrm{LMi}^{\star}$
}

\author{
S. Frandsen ${ }^{1}$, M. Fredslund Andersen ${ }^{1}$, K. Brogaard ${ }^{1}$, C. Jiang ${ }^{2}$, T. Arentoft ${ }^{1}$, F. Grundahl ${ }^{1}$, H. Kjeldsen ${ }^{1}$, \\ J. Christensen-Dalsgaard ${ }^{1}$, E. Weiss ${ }^{1}$, P. Pallé ${ }^{3}$, V. Antoci ${ }^{1}$, P. Kjærgaard ${ }^{4}$, A. N. Sørensen ${ }^{4}$, \\ J. Skottfelt ${ }^{6,5}$, and U. G. Jørgensen ${ }^{5}$ \\ ${ }^{1}$ Stellar Astrophysics Centre, Department of Physics and Astronomy, Aarhus University, Ny Munkegade 120, 8000 Aarhus C, \\ Denmark \\ e-mail: srf@phys.au.dk \\ ${ }^{2}$ School of Physics and Astronomy, Sun Yat-sen University, 2 Daxue Road, Tangjia, Zhuhai, 519082 Guangdong Province, PR \\ China \\ ${ }^{3}$ Instituto de Astrofísica de Canarias (IAC), 38200 La Laguna, Tenerife, Spain \\ ${ }^{4}$ Niels Bohr Institute, University of Copenhagen, Juliane Maries Vej 30, 2100 København Ø, Denmark \\ ${ }^{5}$ Niels Bohr Institute \& Centre for Star and Planet Formation, University of Copenhagen, Øster Voldgade 5, 1350 Copenhagen K, \\ Denmark \\ ${ }^{6}$ Centre for Electronic Imaging, Dept. of Physical Sciences, The Open University, Milton Keynes MK7 6AA, UK
}

Received 17 March 2017 / Accepted 19 February 2018

\begin{abstract}
Context. The Stellar Observation Network Group (SONG) is an initiative to build a worldwide network of $1 \mathrm{~m}$ telescopes with highprecision radial-velocity spectrographs. Here we analyse the first radial-velocity time series of a red-giant star measured by the SONG telescope at Tenerife. The asteroseismic results demonstrate a major increase in the achievable precision of the parameters for red-giant stars obtainable from ground-based observations. Reliable tests of the validity of these results are needed, however, before the accuracy of the parameters can be trusted.

Aims. We analyse the first SONG time series for the star $46 \mathrm{LMi}$, which has a precise parallax and an angular diameter measured from interferometry, and therefore a good determination of the stellar radius. We use asteroseismic scaling relations to obtain an accurate mass, and modelling to determine the age.

Methods. A 55-day time series of high-resolution, high S/N spectra were obtained with the first SONG telescope. We derive the asteroseismic parameters by analysing the power spectrum. To give a best guess on the large separation of modes in the power spectrum, we have applied a new method which uses the scaling of Kepler red-giant stars to $46 \mathrm{LMi}$.

Results. Several methods have been applied: classical estimates, seismic methods using the observed time series, and model calculations to derive the fundamental parameters of $46 \mathrm{LMi}$. Parameters determined using the different methods are consistent within the uncertainties. We find the following values for the mass $M$ (scaling), radius $R$ (classical), age (modelling), and surface gravity (combining mass and radius): $M=1.09 \pm 0.04 M_{\odot}, R=7.95 \pm 0.11 R_{\odot}$ age $t=8.2 \pm 1.9 \mathrm{~Gy}$, and $\log g=2.674 \pm 0.013$.

Conclusions. The exciting possibilities for ground-based asteroseismology of solar-like oscillations with a fully robotic network have been illustrated with the results obtained from just a single site of the SONG network. The window function is still a severe problem which will be solved when there are more nodes in the network.
\end{abstract}

Key words. stars: fundamental parameters - stars: individual: HD 94264 - techniques: radial velocities - telescopes

\section{Introduction}

Asteroseismology has made a big step forward thanks to spaceborne photometry missions. This has been a revolution, particularly for red-giant stars (Bedding et al. 2011; Beck et al. 2012; Christensen-Dalsgaard 2014), as the need for very long time series has been solved by the long-duration observations of CoRoT and Kepler. Ground-based campaigns suffer from much shorter time coverage, and even for long campaigns (Arentoft et al. 2008) we are far from the time coverage of space-borne missions. Furthermore, campaigns must use highprecision radial velocities to detect the stellar oscillations since ground-based photometry is unable to obtain the required precision (Stello et al. 2006, 2007).

\footnotetext{
* Based on observations made with the Hertzsprung SONG telescope operated at the Spanish Observatorio del Teide on the island of Tenerife by the Aarhus and Copenhagen Universities and by the Instituto de Astrofísica de Canarias.
}

However, it is still useful to conduct ground-based radialvelocity campaigns, if we can use dedicated networks of telescopes which can give close to $24 \mathrm{~h}$ coverage for extended periods as discussed in detail by Arentoft et al. (2014). By measuring stellar oscillations using radial velocities the effects of surface granulation are strongly reduced compared to photometric measurements. This allows us to detect modes at lower frequencies, and modes of $l=3$ are easier to detect using radial velocities.

We are particularly interested in nearby stars where an angular diameter can be measured accurately with an interferometer. The idea of combining interferometric measurements with observations of solar-type oscillations in the context of red-giant stars was employed for the star $\epsilon$ Oph (Mazumdar et al. 2009), where the asteroseismic data were obtained from space with the photometric MOST mission (Walker et al. 2003). This was a favourable case because the angular diameter was measured with high accuracy and the space data do not suffer from atmospheric 
and alias problems. This is reflected in the good agreement found between the interferometric radius and the asteroseismic radius. Another similar study has been presented by Beck et al. (2015) for the red-giant stars $\gamma$ Per and $\theta^{1}$ Tau, where the asteroseismic measurements come from multisite radial-velocity measurements.

The Stellar Observations Network Group (SONG) is an initiative which aims to establish a ground-based network of automated $1 \mathrm{~m}$ telescopes to obtain high-precision radial-velocity measurements of nearby, bright stars and to study their properties, primarily using the technique of asteroseismology. The targets being studied will be close enough that accurate parameters, such as angular diameter, parallax, temperature, and abundances, are well known in advance or can be easily determined.

The first SONG node consists of a $1 \mathrm{~m}$ telescope produced by ASTELCO GmBH and is installed at the Observatorio del Teide, Tenerife, Spain. There are two instruments: a lucky imaging photometer (Skottfelt et al. 2015) and a high-resolution spectrograph $(R=100000)$ installed at the Coudé focus in a container next to the dome. The spectrograph, which is the instrument relevant for this paper, has been optimized for high-precision radial-velocity measurements and employs an iodine cell for wavelength reference. All operations are fully automatic and no operator is needed on site or remotely. For more details see Andersen et al. (2014, 2016). The SONG radial-velocity pipeline is described in Grundahl et al. (2017). In this paper we present the results of the first long test run with the SONG node on Tenerife.

\section{The first SONG target $46 \mathrm{LMi}$}

The bright ( $V=3.83$ ) red giant 46 LMi (HR 4247, HD 94264 , HIP 53229) with spectral type K0III was selected as a suitable first target for SONG as the amplitudes and the timescale of the solar-like oscillations in this star are large. These requirements were necessary to test the telescope and spectrograph performance.

The star also presents a good test case for asteroseismology as there are several high-precision measurements of its basic parameters (Table 1). This includes a well-determined parallax $(\pi=34.38 \pm 0.21$ mas, van Leeuwen 2007), and an angular diameter $\left(\Theta_{L}=2.54 \pm 0.03\right.$ mas $)$ measured by interferometry (Nordgren et al. 1999). Together, these lead to a classical measurement of the radius of $R=7.95 \pm 0.11 R_{\odot}$.

In Table 1 the selected values from the literature are listed. The estimate of the age comes from the possible membership of the moving group WOLF 630 (Bubar \& King 2010) of 46 LMi.

\section{Observations and data reduction}

The SONG node permitted a very good time coverage, but with just one node operational the data still suffer from gaps in the time series during daytime. Nevertheless, we were able to obtain a very long time series without involving any observer.

We obtained a total of 3211 exposures, each with an exposure time of $240 \mathrm{~s}$ and a CCD readout time of $2.5 \mathrm{~s}$. The observations were carried out from 14 February to 27 April 2014, with data obtained on 35 of the nights. The main causes for interruptions were poor weather conditions and technical work being done on the telescope and control system. All observations were carried out automatically; in the late afternoon we inserted the observing requests for the coming night into our queue system, and these requests were then executed during the night. All calibration files (bias, flats, ThAr reference) were obtained automatically each afternoon prior to the start of the observations. Fig. 1 shows the

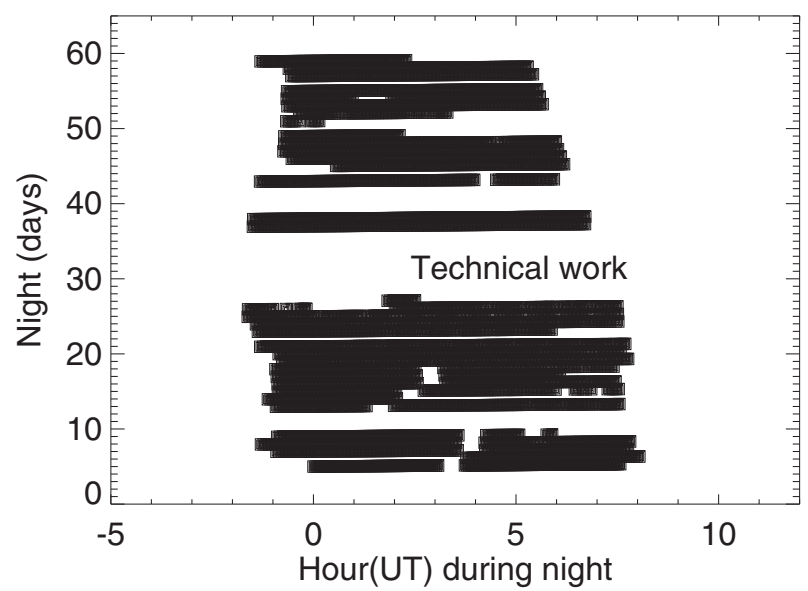

Fig. 1. Time coverage of the 46 LMi campaign. First night: 14 February 2014.

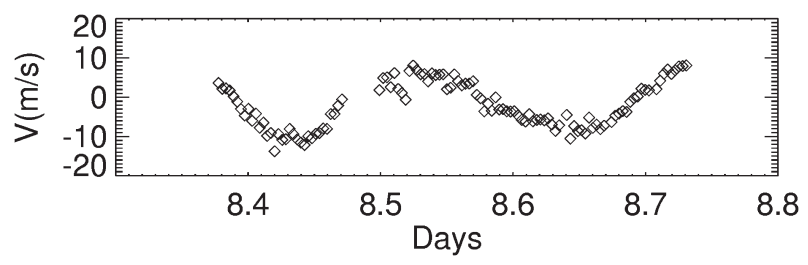

Fig. 2. Radial velocity curve of a single night.

distribution of data points over the 55-day period allocated to this target. The large gap is due to a period of necessary technical upgrades; the data were obtained while the telescope was being commissioned. The rest of the time the only interruptions were due to bad weather.

The spectrograph has six possible slits, and for all observations we used the one providing a resolution of 90000 . For accurate wavelength calibration, we used an iodine cell. All spectra were bias corrected, flat-fielded and wavelength calibrated using the REDUCE package implemented in IDL (see Piskunov $\&$ Valenti 2002) ${ }^{1}$. To extract the precise radial velocities we used the software iSONG (also IDL based) (see e.g. Corsaro et al. (2012), Antoci et al. (2013), and Grundahl et al. (2017) for more details). There are a total of 24 useful orders with iodine lines available for calculating the velocities.

In Fig. 2 we present a full night of the radial-velocity measurements. The typical characteristic timescale of variability of the stochastic oscillations is clearly seen in the velocity curve as the noise level per data point is much lower than the periodic signal. Typically, the instrumental noise per data point is $1.5 \mathrm{~m} \mathrm{~s}^{-1}$ on a good night, where the noise has been calculated as the scatter around a smooth curve through the data.

\section{Analysis of the radial-velocity time series}

\subsection{Frequency of maximum power: $v_{\max }$}

The power spectrum (shown in Fig. 3) was calculated as a fit of sinusoids to the raw radial-velocity time series, as described in Frandsen et al. (1995). The oscillation signal is clearly visible as the power excess around $60 \mu \mathrm{Hz}$. A low-frequency filtering has been applied where ten frequencies below $6 \mu \mathrm{Hz}$ were cleaned from the power spectrum. The effect of single-site observations is illustrated by the window function in the inset, which

1 http://www . astro. uu. se/ piskunov/RESEARCH/REDUCE/ 
Table 1. Basic parameters of $46 \mathrm{LMi}$.

\begin{tabular}{lrc}
\hline \hline Parameter & Measured value & Reference \\
\hline$V$ & 3.83 & SIMBAD Wenger et al. (2000) \\
Temperature $T_{\text {eff }}$ & $4690 \pm 50 \mathrm{~K}$ & Bubar \& King (2010) \\
Metal abundance $[\mathrm{Fe} / \mathrm{H}]$ & $-0.1 \pm 0.1$ & Bubar \& King (2010) \\
Parallax (mas) & $34.38 \pm 0.21$ & van Leeuwen (2007) \\
Angular diameter (mas) & $2.54 \pm 0.03$ & Nordgren et al. (1999) \\
Radius $R / R_{\odot}$ & $7.95 \pm 0.11$ & from parallax and angular diameter \\
Surface gravity log $g$ & $2.61 \pm 0.2$ & Bubar \& King (2010) \\
Luminosity $L / L_{\odot}$ & $27.42 \pm 1.38$ & from $T_{\text {eff } \text { and } R \text { above }}$ \\
Rotation $v \sin (i)$ & $2.1 \mathrm{~km} \mathrm{~s}$ & Massarotti et al. (2008) \\
Possible age (see text) & $2.7 \pm 0.5 \mathrm{~Gy}$ & Bubar \& King (2010) \\
\hline
\end{tabular}

shows strong alias peaks at $\pm 11.57 \mu \mathrm{Hz}$ corresponding to the daily gap in the time series. This significantly complicates the determination of the frequencies of the $p$ modes.

The frequency of maximum power $\left(v_{\max }\right)$ is a widely used value which relates to the large frequency separation through scaling relations (Stello et al. 2009; Huber et al. 2010; Handberg et al. 2017; Arentoft et al. 2017). Based on the available data we determined $v_{\max }$ from a Gaussian fit to the high-pass filtered power spectrum. The uncertainty on $v_{\max }$ was determined by splitting the time series into seven chunks, each with the same number of data points. For each chunk the unfiltered power spectrum was calculated and a value for $v_{\max }$ was determined by fitting a Gaussian to the interval showing power excess, $30 \mu \mathrm{Hz}-100 \mu \mathrm{Hz}$. The uncertainty on the final frequency of maximum power was then given as the standard deviation of the mean error. This procedure yielded $v_{\max }=59.4 \pm 1.4 \mu \mathrm{Hz}$. It is worth mentioning that the expected mode lifetime is of the order of the time span of the data set. This can have an effect on the determined $v_{\max }$, which will be highly influenced by the dominant mode in the power spectrum.

\subsection{Individual frequencies}

We have attempted to determine the frequencies of modes in the power spectrum of $46 \mathrm{LMi}$ using two techniques. In the first method, illustrated in Fig. 4, peaks found in a smoothed power spectrum are plotted in an échelle diagram with frequency modulo $11.574 \mu \mathrm{Hz}$ on the abscissa and $v$ on the ordinate. Then modes are found by looking for patterns similar to the window function in the vertical direction. The size of the symbols represents the amplitude of each detected peak and can be used to select the correct frequency, but in some cases an alias might be obtained instead. Some of the peaks shown in the figure are statistically non-significant and are only included to look for the aliases in the window pattern.

In the second method an iterative frequency determination is performed using PERIOD04. First, the frequency of the mode with maximum signal is found. A weighted cosine fit to the time series with the corresponding period is calculated and subtracted from the time series. This way side bands caused by the window function are removed in the power spectrum. This is repeated on the residual spectrum, but the following iterations simultaneously fit multiple cosines to the time series. This operation is halted when the same number of frequencies as in the first method has been reached. This method is possible because the resolution in the power spectrum $(0.2 \mu \mathrm{Hz})$ is larger than the line width of the stochastic modes in red giants $(\approx 0.1 \mu \mathrm{Hz}$, Handberg et al. 2016). This means excited modes will be represented by
Table 2. Possible modes in $46 \mathrm{LMi}$.

\begin{tabular}{llll}
\hline \hline $\begin{array}{l}v_{1} \\
(\mu \mathrm{Hz})\end{array}$ & $\begin{array}{l}v_{2} \\
(\mu \mathrm{Hz})\end{array}$ & $\begin{array}{l}A_{2}^{1} \\
\left(\mathrm{~m} \mathrm{~s}^{-1}\right)\end{array}$ & $\mathrm{S} / \mathrm{N}^{2}$ \\
\hline 58.986 & 58.958 & 5.1 & 72.9 \\
53.705 & 53.703 & 2.3 & 32.9 \\
64.838 & 64.837 & 1.9 & 27.1 \\
60.919 & 60.914 & 1.9 & 27.1 \\
51.160 & 51.157 & 1.9 & 27.1 \\
64.148 & 64.143 & 1.7 & 24.3 \\
71.345 & $(82.904)$ & - & $\sim 25$ \\
48.796 & $(60.451)$ & - & $\sim 25$ \\
\hline
\end{tabular}

Notes. ${ }^{(1)} v_{1}$ is the frequency from method 1. $v_{2}$ and $A_{2}$ the frequency and amplitude from method 2 (PERIOD4). ${ }^{(2)}$ Noise level calculated at high frequency to $0.07 \mathrm{~m} / \mathrm{s}$ in PERIOD04.

only one peak in the power spectrum in most cases and not by a set producing a Lorentzian profile, which is the case when the observations span many life cycles.

Finally, we get a list of modes (frequency, amplitude, and phase), where modes are only included if they are detected by both methods and agree to better than $0.1 \mu \mathrm{Hz}$. Both results $\left(v_{1}, v_{2}\right)$ are presented in Table 2 to give an idea of the precision, and generally good agreement is seen. The amplitude $A_{2}$ is given for the PERIOD04 method. For two frequencies PERIOD04 detects what could be an alias, which we have enclosed in parentheses. All modes included in Table 2 have high signal-to-noise ratios $(\mathrm{S} / \mathrm{N})$. More modes are likely present; however, for the remaining peaks we were not able to determine which are the true modes and which are the aliases, and they have therefore not been included.

\subsection{Solution using $v_{\max }$}

The global asteroseismic observables include $v_{\max }$ defined above and the large frequency separation $\Delta v$, i.e. the average frequency spacing between modes of the same degree and adjacent orders.

As presented by Miglio et al. (2012), the asteroseismic scaling relations for mass can be expressed in the following four different ways if the luminosity $L$ is included as an independent variable along with $T_{\text {eff }}, \Delta v$, and $v_{\max }$ :

$$
\begin{aligned}
\frac{M}{M_{\odot}} & \simeq\left(\frac{\Delta v}{\Delta v_{\odot}}\right)^{-4}\left(\frac{v_{\text {max }}}{v_{\max , \odot}}\right)^{3}\left(\frac{T_{\text {eff }}}{T_{\text {eff } \odot}}\right)^{3 / 2} \\
\frac{M}{M_{\odot}} & \simeq\left(\frac{\Delta v}{\Delta v_{\odot}}\right)^{2}\left(\frac{L}{L_{\odot}}\right)^{3 / 2}\left(\frac{T_{\text {eff }}}{T_{\text {eff } \odot}}\right)^{-6}
\end{aligned}
$$




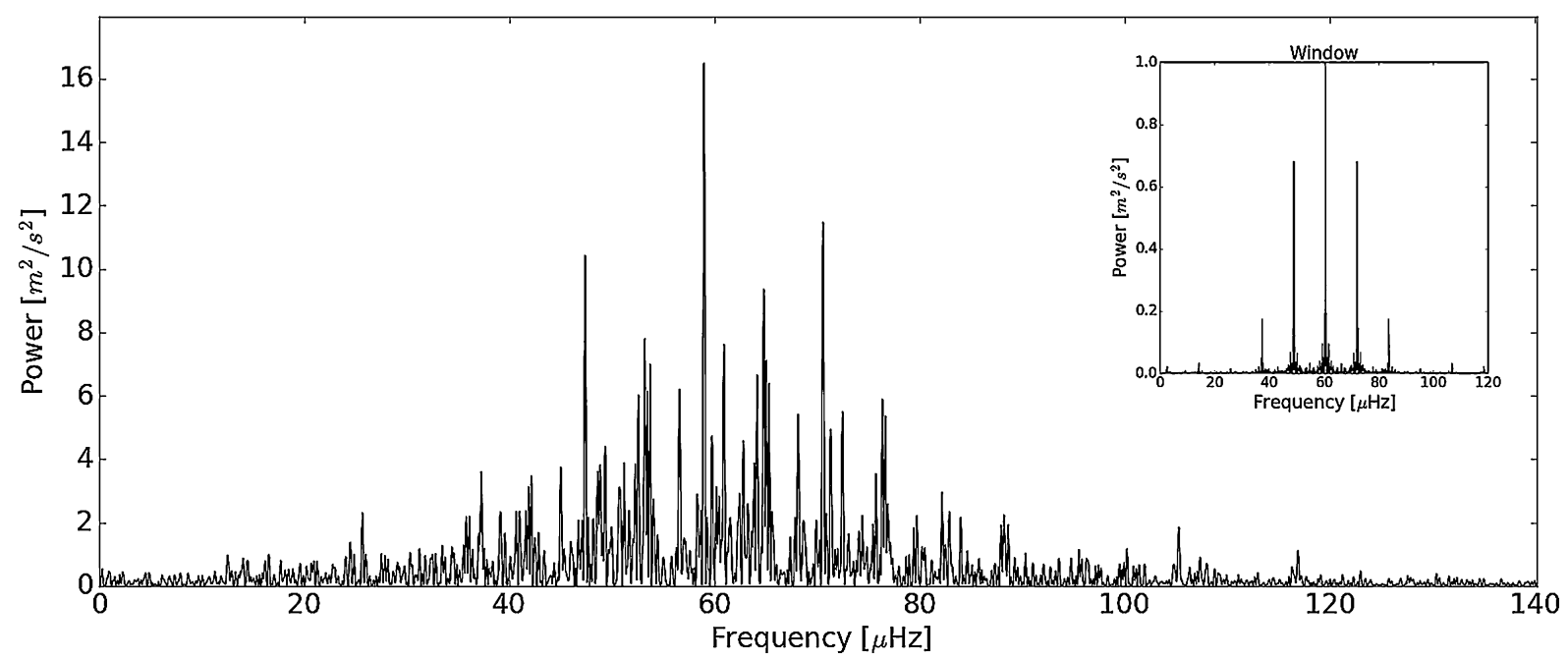

Fig. 3. High-pass filtered power spectrum of $46 \mathrm{LMi}$. The inset shows the normalized window function, which shows strong sidelobes.

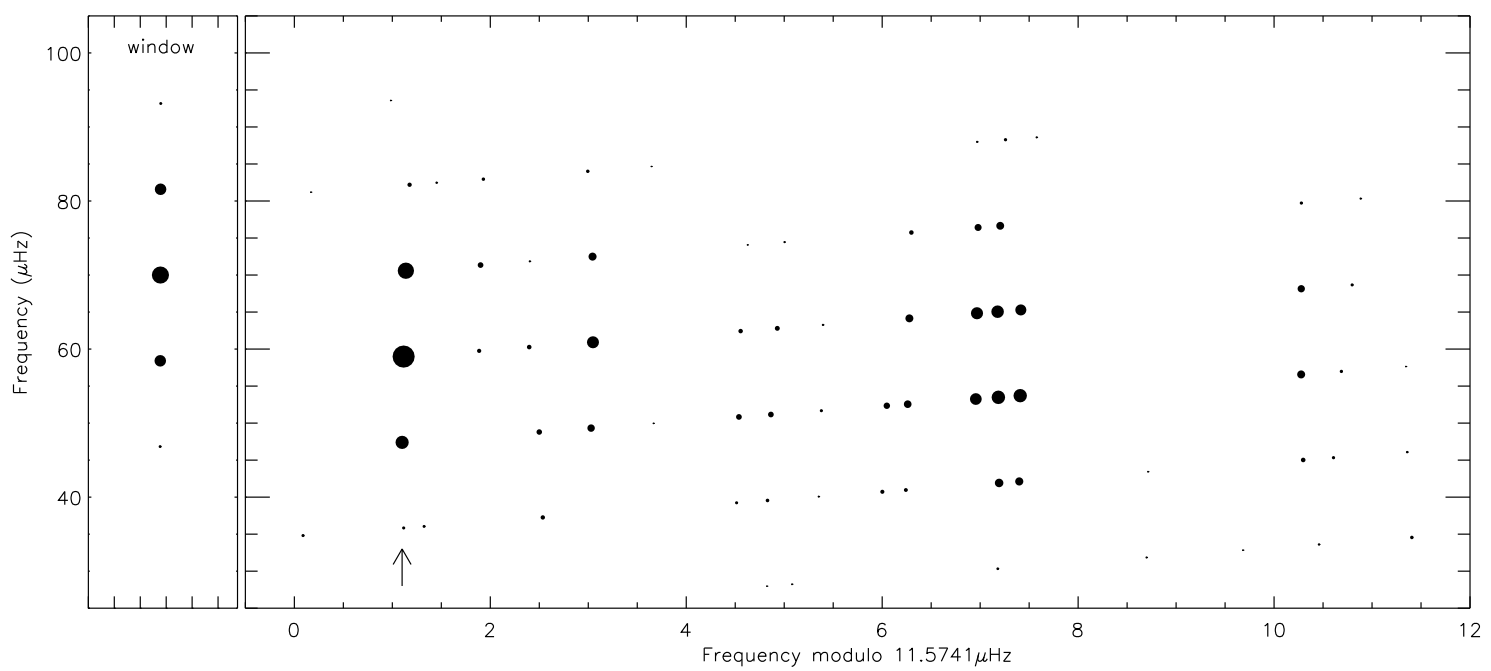

Fig. 4. Échelle diagram with respect to the daily alias $11.57 \mu \mathrm{Hz}$, which brings out peaks belonging to the window function. This is the basis for locating a number of possible modes listed in Table 2. The arrow points to the five peaks corresponding to the dominant mode in the power spectrum.

$\begin{aligned} \frac{M}{M_{\odot}} & \simeq\left(\frac{v_{\text {max }}}{v_{\text {max }, \odot}}\right)\left(\frac{L}{L_{\odot}}\right)\left(\frac{T_{\text {eff }}}{T_{\text {eff } \odot}}\right)^{-7 / 2}, \\ \frac{M}{M_{\odot}} & \simeq\left(\frac{v_{\text {max }}}{v_{\text {max }, \odot}}\right)^{12 / 5}\left(\frac{\Delta v}{\Delta v_{\odot}}\right)^{-14 / 5}\left(\frac{L}{L_{\odot}}\right)^{3 / 10} .\end{aligned}$

Using $L$ we exploit the asteroseismic scaling relation for mass in the form that does not involve $\Delta v$. We use the standard asteroseismic values and $T_{\text {eff }}$ for the Sun (Miglio et al. 2012), which are $\Delta v_{\odot}=135 \mu \mathrm{Hz}, v_{\max , \odot}=3100 \mu \mathrm{Hz}$, and $T_{\text {eff, } \odot}=5777 \mathrm{~K}$.

We adopted the luminosity $\left(L=27.42 \pm 1.38 L_{\odot}\right)$ determined by combining the spectroscopic $T_{\text {eff }}$ and radius, $R=7.95 \pm 0.11 R_{\odot}$ from angular diameter and parallax distance since in that way we avoid making use of a bolometric correction. We checked that the luminosity derived in an alternative way from the parallax $\pi=34.38$ mas, the visual magnitude $V=3.83$, and the bolometric corrections from Casagrande \& VandenBerg (2014), $B C_{V}=-0.384$ and $B C_{V, \odot}=-0.068$, are in agreement with the adopted values to well within the $1 \sigma$ uncertainty.

With this approach we obtained $M=1.09 \pm 0.04 M_{\odot}$, after which reversing any of the other mass equations leads to $\Delta v=6.30 \pm 0.08 \mu \mathrm{Hz}$ with the uncertainties determined by bootstrapping (Feigelson \& Babu 2012). With the estimate of the mass and radius we can determine the surface gravity of $46 \mathrm{LMi}$ to $\log g=2.674 \pm 0.013$.

It is well known that a correction to the observed $\Delta v$ is needed before its use with the asteroseismic scaling relations (Brogaard et al. 2016; Handberg et al. 2017; Miglio et al. 2012; Mosser et al. 2013; Guggenberger et al. 2016). From Fig. 3 in Rodrigues et al. (2017) for a mass of $1.09 M_{\odot}$ and $\nu_{\max }=59.4 \mu \mathrm{Hz}$ in the two cases with $[\mathrm{Fe} / \mathrm{H}]=-0.25$ and $[\mathrm{Fe} / \mathrm{H}]=0.00$ to match the $[\mathrm{Fe} / \mathrm{H}]=-0.1$ of $46 \mathrm{LMi}$ we get a correction factor of 0.966 as the mean of the two solutions. Applying this correction to $\Delta v=6.30 \pm 0.08 \mu \mathrm{Hz}$ we obtain an estimate for the observed $\Delta v=6.09 \pm 0.09 \mu \mathrm{Hz}$. The increase in uncertainty comes from the metallicity dependence of the correction. 


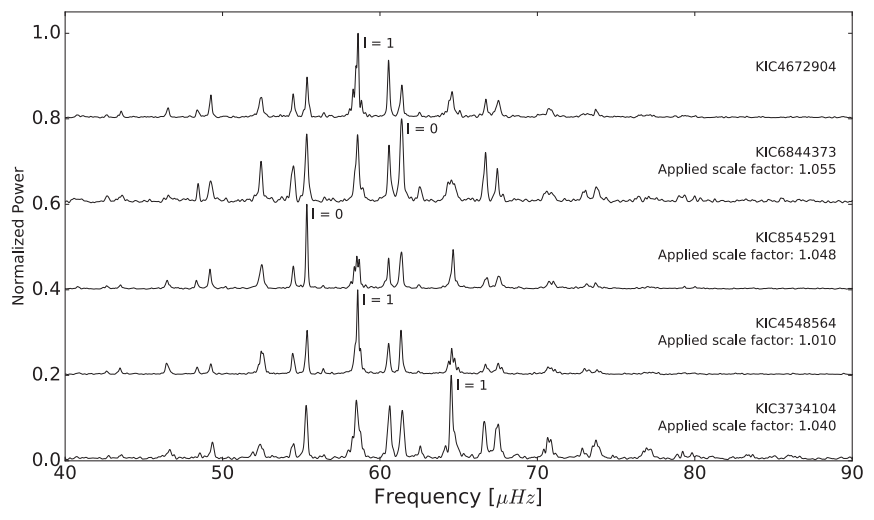

Fig. 5. Four red giants scaled to KIC 4672904. The scale factor applied to the frequency axis of each power spectrum is shown. The 1-mode label marks the mode identification of the dominant mode in the given spectrum.

\subsection{Estimating $\Delta v$ using other methods}

We tried to find the large frequency separation $\Delta v$ by plotting the detected modes in an échelle diagram with different guesses for the large separation, but no convincing $\Delta v$ could be found in this way due to the significant number of alias peaks. Another unsuccessful approach was to calculate the autocorrelation of the power spectrum, and we therefore tried the method that we now discuss here.

We made use of the wealth of information available from the Kepler observations (Jenkins et al. 2010). From the APOKASC ${ }^{2}$ catalogue (Pinsonneault et al. 2014) we chose five red giants observed by Kepler with similar APOKASC effective temperatures and radii to $46 \mathrm{LMi}$ (see Table 3 ). The asteroseismic masses of the five stars are close to the value we determined in Sect. 4.3 for $46 \mathrm{LMi}$. The idea is to apply a scale factor to the power spectrum of a Kepler red giant to match the power spectrum of $46 \mathrm{LMi}$, but first the method was tested on the Kepler stars alone. The frequencies in the power spectrum of each Kepler star was multiplied by a variable scale factor, and the crosscorrelation between the smoothed power spectrum and a chosen smoothed reference spectrum (KIC 4672904) was determined for each value of the scale factor. Each power spectrum was normalized with the power of the highest peak in the region of the oscillation signal. A first guess on the scale factor was based on the $\Delta v$ given in APOKASC and the cross-correlation was done for a range around this value. For each star the maximum in the cross-correlation function was found and this value of the scale factor was applied to the frequencies in the power spectrum. The result of this test (see Fig. 5) demonstrates how well all modes align and proves that the method works for this sample of Kepler stars. We also tested the method on a larger sample of more than 50 Kepler stars which were not as similar to $46 \mathrm{LMi}$, but we still obtained a good agreement.

It is important to mention that applying the determined scale factor for the individual Kepler stars to the $\Delta v$ of the reference does not produce the exact same $\Delta v$ as given in APOKASC but they are well within the $1 \sigma$ uncertainties. The scaled values are given in Table 3.

The method was then applied to $46 \mathrm{LMi}$ which was used as the reference for the cross-correlation. In Fig. 6 the

2 APOKASC comes from a combination of Apache Point Observatory Galactic Evolution Experiment (APOGEE) and of the Kepler Asteroseismic Science Consortium (KASC)

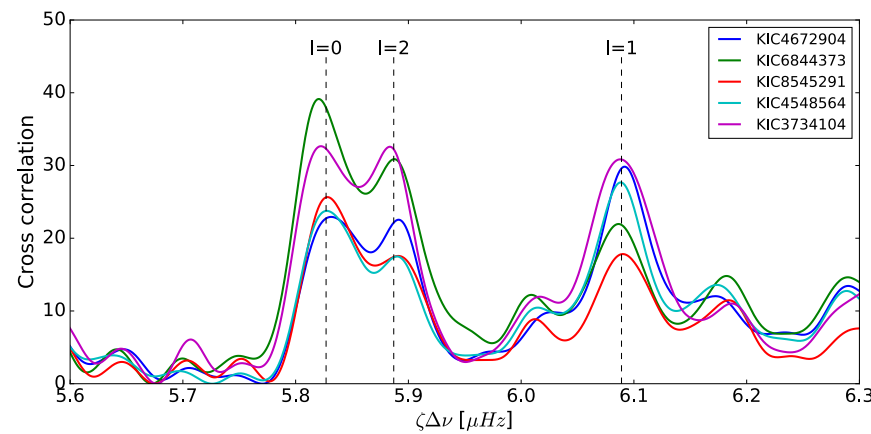

Fig. 6. Cross-correlation function for each of the Kepler stars correlated with $46 \mathrm{LMi}$. The abscissa is the scale factor multiplied by the $\Delta v$ of KIC 4672904. The dashed lines indicate the different $l$-values coming from the identified modes in the Kepler stars when scaled to the dominant mode in $46 \mathrm{LMi}$. See the text for further information.

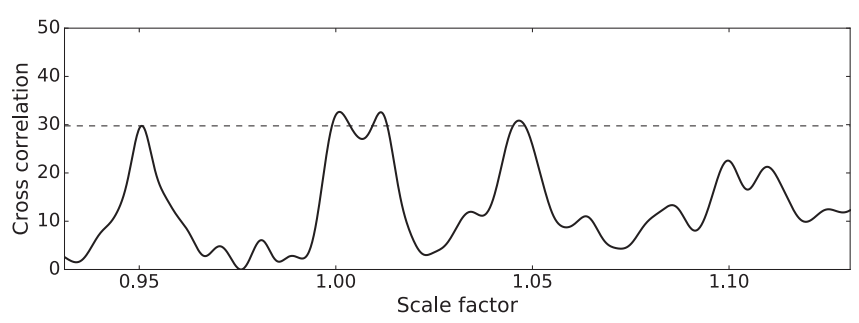

Fig. 7. Cross-correlation between KIC 3734104 and 46LMi for a larger range of scale factors. The peak at 0.95 corresponds to the match when the dominant mode in KIC 3734104 (see bottom panel in Fig. 5) is scaled to the dominant mode in $46 \mathrm{LMi}$

cross-correlation function for each of the Kepler stars from Table 3 is shown. The correlation to $46 \mathrm{LMi}$ is almost entirely dominated by the highest peak in the $46 \mathrm{LMi}$ power spectrum producing a highly correlated signal when matched with a central peak (around $60 \mu \mathrm{Hz}$ in Fig. 5) in the Kepler red giants. This results in three dominant peaks in the cross-correlation corresponding to $l=0,1$, and 2 from the Kepler stars scaled to the dominant peak in 46 LMi. In the case of KIC 8545291 and KIC 3734104 the dominant mode in the power spectrum is outside the central region (see Fig. 5). Nevertheless, it is still one of the modes in the central region that produces the highest correlation. This is illustrated in Fig. 7 where the cross-correlation between KIC 3734104 and $46 \mathrm{LMi}$ is shown with a larger range of scale factors. The first peak in this figure corresponds to the match when the dominant mode in KIC 3734104 is scaled to the dominant mode in $46 \mathrm{LMi}$. Even though this mode is almost twice as high in the power spectrum the cross-correlation is still stronger around the central modes around $60 \mu \mathrm{Hz}$.

The three possible values of $\Delta v$ from the correlation corresponding to the different l-values can be determined with high accuracy (better than $0.01 \mu \mathrm{Hz}$ ), but the real uncertainty on these values comes from the uncertainty in the $\Delta v$ from the APOKASC catalogue. We selected KIC 4672904 as our reference for the scaled $\Delta v$ in Table 3 because it is the one closest to $46 \mathrm{LMi}$ in the physical parameters. These values for $\Delta v$ are used to plot the correlation as a function of frequency on the abscissa in Fig. 6. This is simply done by multiplying the given $\Delta v$ with the scale-factor values found from the cross-correlations. This means that if we selected another star as our reference, the abscissa could shift up to $0.05 \mu \mathrm{Hz}$ (in the case of KIC 6844373).

From this scaling method we get three possible values for the large frequency separation for $46 \mathrm{LMi}$, which we take as the 
Table 3. Large separation of $46 \mathrm{LMi}, \Delta v_{46 \mathrm{LMi}}$ in $\mu \mathrm{Hz}$ from scaling the large separation $\Delta v$ of Kepler giants.

\begin{tabular}{crrrrrrr}
\hline \hline Kepler target & $\Delta v(\mu \mathrm{Hz})^{\mathrm{a}}$ & $\Delta v_{\text {scaled }}(\mu \mathrm{Hz})^{\mathrm{b}}$ & $\Delta v_{46 L \mathrm{Lmi}}(\mu \mathrm{Hz})^{\mathrm{c}}$ & $T_{\text {eff }}(\mathrm{K})^{\mathrm{a}}$ & $M / M_{\odot}{ }^{\mathrm{a}}$ & $R / R_{\odot}^{\mathrm{a}}$ & {$[\mathrm{Fe} / \mathrm{H}]^{\mathrm{a}}$} \\
\hline KIC 4672904 & $6.05 \pm 0.13$ & - & $5.82,5.89,6.09$ & 4614 & $1.11 \pm 0.11$ & 8.19 & -0.38 \\
KIC 6844373 & $5.78 \pm 0.15$ & 5.73 & $5.87,5.94,6.14$ & 4556 & $1.10 \pm 0.15$ & 8.41 & -0.05 \\
KIC 8545291 & $5.81 \pm 0.15$ & 5.78 & $5.86,5.93,6.13$ & 4522 & $1.09 \pm 0.13$ & 8.34 & -0.13 \\
KIC 4548564 & $5.98 \pm 0.13$ & 5.99 & $5.84,5.91,6.11$ & 4503 & $1.05 \pm 0.12$ & 8.09 & -0.33 \\
KIC 3734104 & $5.85 \pm 0.14$ & 5.82 & $5.86,5.93,6.13$ & 4514 & $1.21 \pm 0.17$ & 8.60 & 0.02 \\
\hline
\end{tabular}

Notes. ${ }^{(a)}$ Values from Pinsonneault et al. (2014); $T_{\text {eff }}$ and [Fe/H] are the UNCORRECTED ASPCAP (G1). ${ }^{(b)}$ Scaled from KIC 4672904. ${ }^{(c)}$ Scaled from the given Kepler star based on the $l=0,2$, and 1 maximum in the correlation function, respectively.

mean of the five scaled $\Delta v$ from Table 3 with the uncertainty given as the half of the full range in these values:

$\Delta v_{46 L M i}=(5.85 \pm 0.03) \mu \mathrm{Hz}$ (Dominant mode in 46 LMi is $\left.l=0\right)$ $\Delta v_{46 L M i}=(5.92 \pm 0.03) \mu \mathrm{Hz}$ (Dominant mode in 46 LMi is $\left.l=2\right)$ $\Delta v_{46 L M i}=(6.12 \pm 0.03) \mu \mathrm{Hz}$ (Dominant mode in 46 LMi is $l=1$ )

We cannot say which $\Delta v$ is the correct one because we do not know the identification of the dominant mode in $46 \mathrm{LMi}$, but by looking at each value we can argue which are more or less likely. Remembering the value of $v_{\max }$ found in Sect. 4.3, and using this with the known physical parameters of $46 \mathrm{LMi}$ and the asteroseismic scaling relations, we found $\Delta v=6.09 \pm 0.09 \mu \mathrm{Hz}$. From this, we can argue that the $\Delta v$ corresponding to the highest peak being a $l=1$ mode is the most likely. This solution has some implications which do not support it. When the power spectrum from any of the Kepler stars is scaled using the $l=1$ value, none of their radial modes near $v_{\max }$ match peaks in the power spectrum of $46 \mathrm{LMi}$. This seems unlikely since no Kepler red giant in our sample shows this behaviour. However, it is well known that radial modes have less power relative to the dipole modes in RV compared to intensity (Bedding et al. 1996), which again could favor this solution. The relative ratio between $l=0$ and $l=1$ modes for SONG was computed in Handberg \& Campante (2011) and was determined to be 1.35. The solution where the dominant mode in $46 \mathrm{LMi}$ is a $l=0$ mode is less likely $(\sim 3 \sigma)$ when compared to the $\Delta v$ estimated from $v_{\max }$ in Sect. 4.3. However, more modes from the power spectrum of a given Kepler giant match peaks in the power spectrum of $46 \mathrm{LMi}$. This could be a result of the $\Delta v$ being very close to one half of the daily splitting produced by the sampling from single-site observations. Every second mode in the Kepler power spectrum would then roughly match an alias peak if this were the true value and the high correlation for the $l=0$ mode might simply be a result of the sampling of our data.

The same is the case for the value related to the dominant mode in $46 \mathrm{LMi}$ being a $l=2$ mode. This value is also close to one half of the daily alias. The fact that it is a bit further away from the alias peaks might mirror the correlation power in Fig. 6, which is in general a bit lower for the $l=2$ peaks than the $l=0$ modes.

The high peaks in Fig. 6 have their origin in the dominant modes in the power spectra of $46 \mathrm{LMi}$ and the template. To reduce the effect of the dominant modes we have divided the power spectra by a Gaussian fit to the power spectra before cross correlation. We have also performed a test with a power law. In none of the cases did we see any significant change of the results. The heights of the peaks in Fig. 6 were modified, but not the positions.

All three values of $\Delta v$ can be used to scale a given Kepler star to $46 \mathrm{LMi}$, and all three fail to match all frequencies given in Table 2 . This indicates that we have either incorrectly determined
Table 4. Most likely model parameters given the constraints from observations.

\begin{tabular}{lrr}
\hline \hline Parameter & Value & $1 \sigma$ error \\
\hline$M / M_{\odot}$ & 1.09 & 0.03 \\
$Z$ & 0.017 & 0.003 \\
$\alpha$ & 2.07 & 0.11 \\
$R / R_{\odot}$ & 7.95 & 0.13 \\
$T_{\text {eff }}(\mathrm{K})$ & 4688 & 86 \\
$L / L_{\odot}$ & 27.3 & 2.2 \\
Age $(\mathrm{Gyr})$ & 8.2 & 1.9 \\
$\log g$ & 2.67 & 0.01 \\
$\nu_{\max }(\mu \mathrm{Hz})$ & 59.4 & 2.0 \\
$\Delta v(\mu \mathrm{Hz})$ & 6.3 & 0.1 \\
\hline
\end{tabular}

Notes. See the text for explanation of the errors given.

some of the frequencies given or that none of the stars in our sample matches 46 LMi completely, or a combination of the two. We know from the Kepler red giants that some show clear splitting of the dipole modes caused by mixing with g-modes. If this were the case in $46 \mathrm{LMi}$ it would make the analysis of the power spectrum significantly more difficult since all split modes would produce alias peaks due to the window function.

\section{Modelling}

We can show that a model exists that reproduces our new asteroseismic results and existing non-seismic measurements. In addition, we can find a "best" solution in terms of all constraints.

We employed the ASTEC evolution code (Christensen-Dalsgaard 2008b) for stellar evolution modelling computations, and the ADIPLS oscillation package (Christensen-Dalsgaard 2008a) for frequency calculations. ASTEC can evolve models up to the tip of the red giant branch, which is adequate for $46 \mathrm{LMi}$. The input physics of the ASTEC version that we used included the latest OPAL opacity tables (Iglesias \& Rogers 1996), OPAL 2005 equation of state (Rogers \& Nayfonov 2002), and NACRE reaction rates (Angulo et al. 1999). At low temperatures, opacities are obtained from Ferguson et al. (2005). Convection is treated under the assumption of mixing length theory (Böhm-Vitense 1958). We did not take rotation, diffusion, or convective overshoot into consideration in our calculation.

For model selection, we used a tool called DIAMONDS that performs Bayesian parameter estimation and model comparison by means of the nested sampling Monte Carlo (NSMC) algorithm (Corsaro \& De Ridder 2014). The mass, heavy-element abundance $Z$, and mixing-length parameter $\alpha$ are set as free parameters. The mass is restricted to the range of $0.95-1.25 M_{\odot}$, 
$Z$ to the range $0.001-0.03$, and $\alpha$ to $1-3$. Based on a Galactic chemical-evolution model (Carigi 2000; Pietrinferni et al. 2004) the hydrogen abundance $X$ is obtained as $X=-2.4 Z+0.748$, which gives $X=0.7$ when $Z=0.02$ and a helium-to-metal enhancement ratio $\Delta Y / \Delta Z=1.4$.

We use the observed parameters (with their uncertainties) $R, T_{\text {eff }}$ from Table 1, and $v_{\max }$ from Sect. 4.1 as constraints to obtain the likelihood of a given model based on the $\chi^{2}$ value. The results are given in Table 4 where the errors are given as Bayesian errors which only represent the capability of the model to fit the given observations. We use a $68.3 \%$ probability for the Bayesian credible intervals.

If we assume that we know $R$ and $T_{\text {eff }}$, we can use the scaling relation for $v_{\max }$ to calculate $M$ which we did in Sect. 4.3. Not surprisingly, the best fitting model therefore has parameters consistent with the previous results in this paper. The $\chi^{2}$ of the best models is 1.36. The values given in Table 4 are from the Bayesian and they give $\chi^{2}=0.87$. Removing the constraint on $v_{\max }$ changes the results very little. The age determined here is significantly older than found by Bubar \& King (2010), but it is the expected age for $\mathrm{a} \sim 1.09 M_{\odot}$ red giant. If $46 \mathrm{LMi}$ were indeed a member of the moving group Wolf 630, this would suggest a higher age for the group. More likely, 46 LMi is not a member.

\section{Conclusions}

Even if SONG is not yet a network of telescopes, the use of just one dedicated automatic telescope leads to ground-based results comparable to or better than previous asteroseismic campaigns. The reason is the easy way SONG can perform long time observations. The main results are summarized here.

- The first SONG observations demonstrate that we have perhaps obtained the best ground-based red-giant power spectrum to date. $46 \mathrm{LMi}$ shows a clear solar-like power excess centred at $59.4 \pm 1.4 \mu \mathrm{Hz}$, where the uncertainty is mainly due to the stochastic excitation. The power reaches nearly zero at low frequencies $(\leq 30 \mu \mathrm{Hz})$ for the high-pass filtered radial-velocity time series. The power spectrum is affected by alias peaks due to the use of single-site data. Comparison to stars observed by Kepler leads to three possible values for the large frequency separation. We therefore base our mass and radius estimates on $v_{\max }$ as the only asteroseismic measure and combine it with classical results, as described in Sect. 4.3.

- Our best estimate of the mass of $46 \mathrm{LMi}$ (from the $v_{\max }$ solution) is $M=1.09 \pm 0.04 M_{\odot}$ and the radius from classical estimates is $R=7.95 \pm 0.11 R_{\odot}$. The surface gravity estimate is $\log g=2.674 \pm 0.013$ derived from the mass and radius just listed leading to an improved accuracy compared to the spectroscopic gravity $\log g=2.61 \pm 0.2$.

- The age of $8.2 \pm 1.9 \mathrm{Gyr}$ that we find for $46 \mathrm{LMi}$ is significantly higher than that reported by Bubar \& King (2010). This suggests that $46 \mathrm{LMi}$ is not a member of the moving group WOLF 630. We thus demonstrate that asteroseismic age determination can be used to determine membership of stellar constellations such as moving groups and clusters.

Improvements will be possible from multisite observations, as we will be able to identify individual mode frequencies since alias peaks will be highly suppressed. This will lead to a more precise age estimate, and a measurement of mass and radius from the asteroseismic scaling relations that is independent of the distance. We will then be able to get a high-accuracy test of the asteroseismic scaling relations by comparing the radius derived from asteroseismology to that obtained from interferometry. This clearly shows why more SONG nodes are needed.

Acknowledgements. This research took advantage of the Simbad and Vizier databases, operated at the CDS, Strasbourg (France), and NASA's Astrophysics Data System Bibliographic Services. We would like to acknowledge the Villum Foundation, The Danish Council for Independent Research I Natural Science and the Carlsberg Foundation for the support on building the SONG prototype on Tenerife. K.B. acknowledges support from the Villum Foundation. The Stellar Astrophysics Centre is funded by The Danish National Research Foundation (Grant DNRF106) and research was supported by the ASTERISK project (ASTERo-seismic Investigations with SONG and Kepler) funded by the European Research Council (Grant agreement n. 267864). We also gratefully acknowledge the support from the Spanish Ministry of Economy Competitiveness (MINECO) grant AYA2016-76378-P.

\section{References}

Andersen, M. F., Grundahl, F., Christensen-Dalsgaard, J., et al. 2014, in Rev. Mex. Astron. Astrofis., 45, 83

Andersen, M. F., Grundahl, F., Beck, A. H., \& Pallé, P. 2016, in Rev. Mex. Astron. Astrofis., 48, 54

Angulo, C., Arnould, M., Rayet, M., et al. 1999, Nucl. Phys. A, 656, 3

Antoci, V., Handler, G., Grundahl, F., et al. 2013, MNRAS, 435, 1563

Arentoft, T., Kjeldsen, H., Bedding, T. R., et al. 2008, ApJ, 687, 1180

Arentoft, T., Tingley, B., Christensen-Dalsgaard, J., et al. 2014, MNRAS, 437, 1318

Arentoft, T., Brogaard, K., Jessen-Hansen, J., et al. 2017, ApJ, 838, 115

Beck, P. G., Montalban, J., Kallinger, T., et al. 2012, Nature, 481, 55

Beck, P. G., Kambe, E., Hillen, M., et al. 2015, A\&A, 573, A138

Bedding, T. R., Kjeldsen, H., Reetz, J., \& Barbuy, B. 1996, MNRAS, 280, 1155

Bedding, T. R., Mosser, B., Huber, D., et al. 2011, Nature, 471, 608

Böhm-Vitense, E. 1958, Z. Astrophys., 46, 108

Brogaard, K., Jessen-Hansen, J., Handberg, R., et al. 2016, Astron. Nachr., 337, 793

Bubar, E. J., \& King, J. R. 2010, AJ, 140, 293

Carigi, L. 2000, Rev. Mex. Astron. Astrofis., 36, 171

Casagrande, L., \& VandenBerg, D. A. 2014, MNRAS, 444, 392

Christensen-Dalsgaard, J. 2008a, Ap\&SS, 316, 113

Christensen-Dalsgaard, J. 2008b, Ap\&SS, 316, 13

Christensen-Dalsgaard, J. 2014, Asteroseismology of red giants, P. L. Pallé, \& C. Esteban (Cambridge University Press)

Corsaro, E., \& De Ridder, J. 2014, A\&A, 571, A71

Corsaro, E., Grundahl, F., Leccia, S., et al. 2012, A\&A, 537, A9

Feigelson, E., \& Babu, G. 2012, Modern Statistical Methods for Astronomy (Cambridge University Press)

Ferguson, J. W., Alexander, D. R., Allard, F., et al. 2005, ApJ, 623, 585

Frandsen, S., Jones, A., Kjeldsen, H., et al. 1995, A\&A, 301, 123

Grundahl, F., Fredslund Andersen, M., Christensen-Dalsgaard, J., et al. 2017, ApJ, 836, 142

Guggenberger, E., Hekker, S., Basu, S., \& Bellinger, E. 2016, MNRAS, 460, 4277

Handberg, R., \& Campante, T. L. 2011, A\&A, 527, A56

Handberg, R., Miglio, A., Brogaard, K., Bossini, D., \& Elsworth, Y. P. 2016, Astron. Nachr., 337, 799

Handberg, R., Brogaard, K. F., Miglio, A., et al. 2017, MNRAS, 472, 979

Huber, D., Bedding, T. R., Stello, D., et al. 2010, ApJ, 723, 1607

Iglesias, C. A., \& Rogers, F. J. 1996, ApJ, 464, 943

Jenkins, J. M., Caldwell, D. A., Chandrasekaran, H., et al. 2010, ApJ, 713, L120

Massarotti, A., Latham, D. W., Stefanik, R. P., \& Fogel, J. 2008, AJ, 135, 209

Mazumdar, A., Mérand, A., Demarque, P., et al. 2009, A\&A, 503, 521

Miglio, A., Brogaard, K., Stello, D., et al. 2012, MNRAS, 419, 2077

Mosser, B., Michel, E., Belkacem, K., et al. 2013, A\&A, 550, A126

Nordgren, T. E., Germain, M. E., Benson, J. A., et al. 1999, AJ, 118, 3032

Pietrinferni, A., Cassisi, S., Salaris, M., \& Castelli, F. 2004, ApJ, 612, 168

Pinsonneault, M. H., Elsworth, Y., Epstein, C., et al. 2014, ApJS, 215, 19

Piskunov, N. E., \& Valenti, J. A. 2002, A\&A, 385, 1095

Rodrigues, T. S., Bossini, D., Miglio, A., et al. 2017, MNRAS, 467, 1433

Rogers, F. J., \& Nayfonov, A. 2002, ApJ, 576, 1064

Skottfelt, J., Bramich, D. M., Hundertmark, M., et al. 2015, A\&A, 574, A54

Stello, D., Arentoft, T., Bedding, T. R., et al. 2006, MNRAS, 373, 1141

Stello, D., Bruntt, H., Kjeldsen, H., et al. 2007, MNRAS, 377, 584

Stello, D., Chaplin, W. J., Basu, S., Elsworth, Y., \& Bedding, T. R. 2009

MNRAS, 400, L80

van Leeuwen, F. 2007, A\&A, 474, 653

Walker, G., Matthews, J., Kuschnig, R., et al. 2003, PASP, 115, 1023

Wenger, M., Ochsenbein, F., Egret, D., et al. 2000, A\&AS, 143, 9 